\title{
Physics motivations and expected performance of the CMS muon system upgrade with triple-GEM detectors
}

\author{
Rosamaria Venditti ${ }^{* \dagger}$ \\ University and INFN, Bari \\ E-mail: rosamaria.venditti@cern.ch
}

For the LHC High Luminosity phase (HL-LHC) the CMS GEM Collaboration is planning to install new large size triple-GEM detectors in the forward region of the muon system $(1.5<|\eta|<2.2)$ of the CMS detector. The muon reconstruction with triple-GEM chambers information included have been successfully integrated in the official CMS software, allowing physics studies to be carried out. The new sub-detector will be able to cope the extreme particle rates expected in this region along with a high spatial resolution. The resulting benefit in terms of triggering and tracking capabilities has been studied: the expected improvement in the performance of the muon identification and track reconstruction as well as the expected improvement coming from the lowering of the muon $p_{T}$ trigger tresholds will be presented. The contribution will review the status of the CMS upgrade project with the usage of GEM detector, discussing the trigger, the muon reconstruction performance and the impact on the physics analyses.

The European Physical Society Conference on High Energy Physics

22-29 July 2015

Vienna, Austria

* Speaker.

${ }^{\dagger}$ on behalf of the CMS GEM Collaboration 


\section{Towards HL-LHC}

CERN began planning to increase the luminosity of the LHC [1] above the original design even before the machine went into operation. This will undergo a period of machine maintenance (long shutdown, referred to LS1, LS2, LS3), during which all the detectors will adjust their performance to cope the new machine conditions, interleaved to periods of data-taking (referred to as Run-II, Run-III, Run-IV). During the second long shutdown (LS2) planned for mid-2019, the peak luminosity could reach $2 \times 10^{34} \mathrm{~cm}^{-2} \mathrm{~s}^{-1}$, providing an integrated luminosity of over $300 \mathrm{fb}^{-1}$. By 2023 there will be another long shutdown, LS3, that will further improve the LHC machine performance, producing a significant increase in the LHC luminosity. The high luminosity period that will follow LS3 is referred to as HL-LHC or Phase-II. The proposed operating scenario is to level the instantaneous luminosity up to peak value of $5 \times 10^{35} \mathrm{~cm}^{-2} \mathrm{~s}^{-1}$, and to deliver $250 \mathrm{fb}^{-1}$ per year for a further 10 years of operation. Under these conditions the number of pile-up interactions (PU) superimposed to the hard scatter, will rise substantially (on average 50 interactions during Run III and 140 during Run IV) thus becoming a major challenge for the event reconstruction. Moreover, the increase of the integrated radiation dose will lead to detector performance degradation that will need to be addressed. The CMS collaboration has planned an upgrade of the existing detector: during LS3 the experiment will be equipped with an extended tracker, high-granularity calorimeter, and new detectors in the forward region of the muon system [2].

\section{The CMS Muon System Upgrades with GEM detectors}

The CMS muon system[3] is designed to fulfill three main functions: robust and redundant muon identification, muon trigger capabilities and muon momentum measurement. Precision measurements are provided by Drift Tubes (DT) in the barrel, covering acceptances up to $|\eta|<1.2$ and Cathode Strip Chambers (CSC) in the endcaps covering $1.0<|\eta|<2.4$. Resistive Plate Chambers (RPC) cover the region up to $|\eta|<1.6$. The region $|\eta|>1.6$ is only instrumented with CSC. One of the main goals during the upgrade phases will be to restore the originally foreseen redundancy in the forward region beyond $|\eta|>1.6$. The new detectors have to satisfy a high rate capability, $O\left(M H z / \mathrm{cm}^{2}\right)$, a good time resolution for triggering, and a good spatial resolution, $O(100 \mu \mathrm{m})$, for tracking. The gaseous electron multipliers (GEMs) [4], appears to be the optimal solutions, since they fulfill all of these requirements. Indeed, from recent test performed by the CMS GEMs collaboration, it emerges that those detectors are able to sustain rates up to $O\left(10^{5} \mathrm{kHz} / \mathrm{cm}^{2}\right)$, while joining excellent tracking capabilities, thanks to a spatial resolution $\sim 276 \mu \mathrm{m}$, with high time resolution $\sim 4$ ns [5]. The baseline of the CMS GEM project is sketched in Fig. 1. During the LS2, 36 double-layered triple-GEM chambers, called the GE1/1 system, will be installed in front of the first CSC station (ME1/1). The chambers will provide full coverage in $\phi$ and $1.55<|\eta|<2.18$ in pseudo-rapidity. During LS3, two rings of triple-GEM chambers covering up to $1.6<|\eta|<2.45$ will be installed. Each GE2/1 chamber spans about 20 degrees in $\phi$. Finally, a muon near-tagger (ME0) with 18 six-layered triple-GEMs, will cover the region $2.0<|\eta|<3.0$ in pseudo-rapidity, behind the future shortened hadron calorimeter. The geometry of both GE2/1 and ME0 is yet to be finalized and the R\&D activity is still ongoing. 


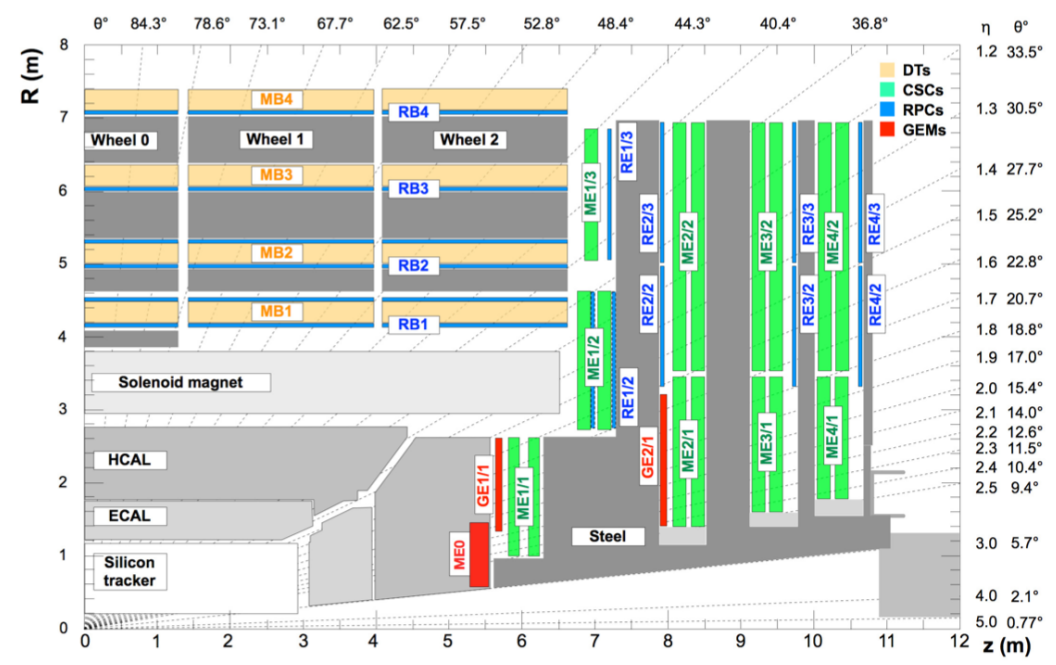

Figure 1: A quadrant of the muon system, showing DT chambers (yellow), RPC (light blue), and CSC (green). The new GEM detector stations are indicated in red (ME0, GE1/1, and GE2/1).

\section{Forward Muon System Upgrade Motivations}

The forward region of the present CMS muon system is characterized by low magnetic field while suffering of large neutron-induced background. In the first station of the muon system, the magnetic field is strong enough to provide a decent bending. In addition, as already discussed in section 2, the region $|\eta|>1.6$ suffers by lacks of redundancy being only instrumented with CSC. For the reasons above, the contribution to the trigger rate coming from the forward region is particularly large. The inclusion of new detectors, with very high spatial and time resolution, could allow a better estimation of the muon transverse momentum $\left(p_{T}\right)$ both for online (L1) trigger and high-level-trigger (HLT) while retaining a good background rejection. As a consequence, all the physics channels characterized by soft muon signatures, such as $H \rightarrow Z^{*} Z \rightarrow 4 \mu$ and $H \rightarrow \tau_{\mu} \tau_{\text {had }}$ would benefit from the lowering of the muon $p_{T}$ thresholds both at L1 and HLT.

\section{Forward Muon System Trigger Challanges}

The muon trigger is the most difficult in the forward region where the bending of the track of the muon candidate in the magnetic field is small, the background is large and the redundancy is poor. These factors lead to muon momentum mis-measurements associated with the tails in the $p_{T}$ resolution of the muon trigger. To reduce the trigger rate, while keeping high efficiency, the new muon detectors should measure more precisely the bending angle in the first muon station, characterized by higher magnetic field. The installation of GE1/1 in front of ME1/1 can improve the $p_{T}$ measurement, thanks to the increased "lever arm" (by a factor of 2.4-3.5) between GEM and CSC stations. The blue curve in Fig. 2 left shows that an efficient single muon trigger (with a plateau efficiency of 94\%) would require a too-high-bandwidth allocation, i.e. $20 \mathrm{kHz}$ out of the total $\mathrm{L} 1$ trigger acceptance rate of $100 \mathrm{kHz}$. Tightening the muon selection criteria will quickly deteriorate the trigger efficiency. Instead, combining the information provided by CSC and GEM, it is possible to develop a combined local trigger that allows to keep or reduce the $p_{T}$ thresholds applied at L1 
to the muon candidate, without efficiency loss, thanks to the improved $p_{T}$ resolution. The purple curve on Fig. 2 left shows that the GEM-CSC local trigger reduces by a factor 10 the single muon trigger rate. In Fig. 2 right it is shown the fast deterioration of the single muon trigger efficiency with even a moderate fraction of non-triggering CSC chambers: the extra measurement points coming from GEM detectors are able to recover possible CSC failures, restoring the redundancy in the forward region and will also yield a higher efficiency, even at very high pile-up scenarios.
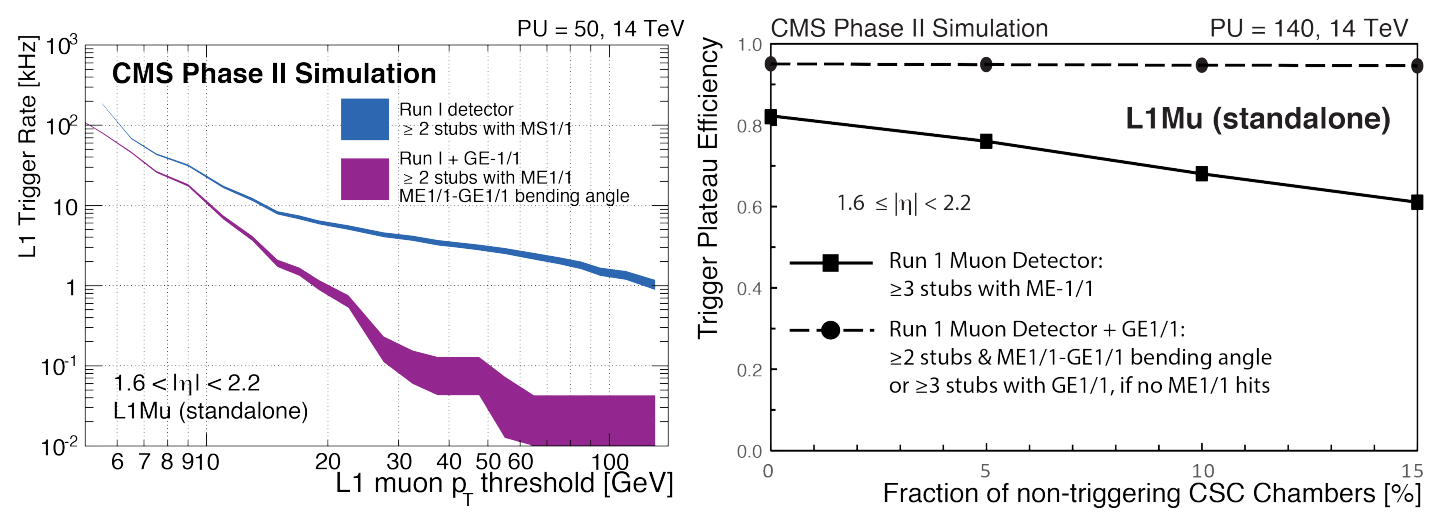

Figure 2: Left: L1 muon trigger rate at a luminosity of $2 \times 10^{34} \mathrm{~cm}^{-2} \mathrm{~s}^{-1}$ as a function of $p_{T}$ threshold. With the addition of GE1/1, the bending angle between ME1/1 and GE1/1 stations can be used and the trigger rate is greatly reduced. Right: single-muon trigger efficiency at the plateau in $p_{T}$ as a function of the fraction of non-triggering CSC chambers.

\section{Muon Reconstruction Performance}

The lack of redundancy of the muon system in the forward region will become an issue if any CSC chambers become inoperative. Fig. 3 shows the standalone muon performance, for a 140 pileup scenario, when ME1/1 is completely broken, with (green markers) and without (black markers) the additional hits provided by including GE1/1 in the standalone muon reconstruction. The same is also shown when ME1/1 is fully operative and the hits from GE1/1 are included (red markers) and are not (blue markers) in the standalone muon fit. The upgraded system shows an overall increase in the reconstruction efficiency (Fig. 3 left) and a reduction in the rate with which the background is misidentified as signal muons (Fig. 3 right). Additional redundancy associated with the GE1/1 system allows the recovery of most of the efficiency losses even in the most pessimistic scenario where the entire ME1/1 becomes inoperable. As already discussed in section 4, with the addition of GE1/1, the bending angle between ME1/1 and GE1/1 stations improves the muon momentum estimate at L1. The same applies for the offline reconstruction. To quantify the impact of the CSC detector degradation on the standalone muon momentum resolution and charge misidentification, we study the relative resolution of the muon curvature measurement. The specific figure of merit chosen is the residual distribution $q / p_{T}$ defined as:

$$
\frac{\delta\left(\frac{q}{p_{T}}\right)}{\frac{q}{p_{T}}}=\frac{\left(q / p_{T}\right)^{r e c}-\left(q / p_{T}\right)^{\operatorname{sim}}}{\left(q / p_{T}\right)^{\operatorname{sim}}}
$$



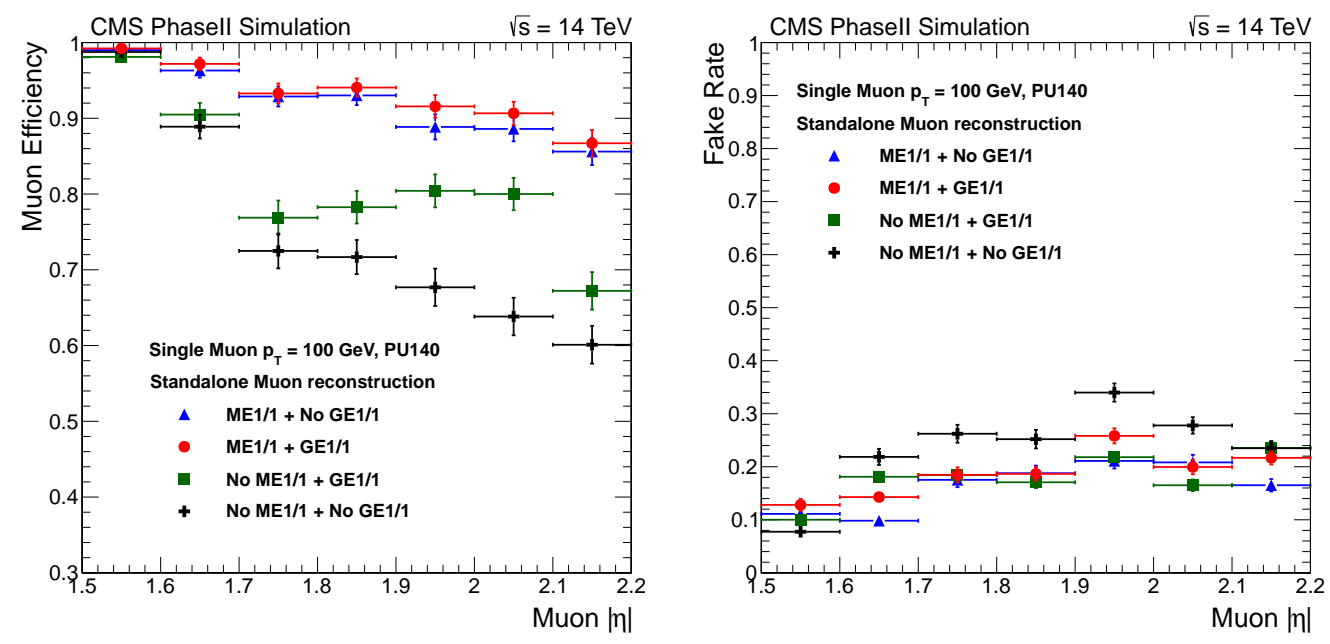

Figure 3: Left: Standalone muon reconstruction efficiency as a function of muon pseudorapidity in case of ME1/1 failure. Right: the corresponding level of fake-rate . The results have been produced by considering event with 140 pile-up interactions. The reconstruction efficiency is recovered by adding GE1/1 while the fake-rate level is kept as low as ME1/1 is fully operational.

where $q$ is the muon charge and sim and rec indicate the simulated and reconstructed quantities. The presence of GE1/1 allows a significant reduction of otherwise unavoidable dramatic deterioration of momentum resolution in the scenario where ME1/1 becomes inoperable. Fig. 4 makes this observation clear by showing the standard deviation $\sigma$ of the distributions for several scenarios as a function of the simulated muon pseudorapidity. Standalone muon reconstruction performance are crucial not only as the first step of global muon reconstruction, but also because many exotic physics channels, with long-lived particles decaying to muons out of the tracker volume, completely relies on it. The demonstrated recovery of the efficiency should be considered as a lower bound to the potential improvement, as the current implementation does not reconstruct GE1/1 segments, and therefore GE1/1 is not used in seeding the standalone reconstruction.

\section{Conclusions and Future perspectives}

The present scope of the CMS GEM project proposes the installation of 3 new stations: GE1/1, GE2/1 in the presently vacant positions in front ME1/1 and ME2/1 and a near-tagger, ME0 in the space left by the future calorimeter. Triple-GEMs provide a suitable solution for the CMS muon trigger and tracking needs in the HL-LHC era thanks to their excellent spatial resolution (276 $\mu \mathrm{m})$ and time resolution (4 ns) and the high-rate capability. Triple-GEM detectors installation in the CMS high eta region will restore the lack of redundancy and allow to improve the muon momentum resolution, measuring more precisely the bending angle and thus helping to reduce the trigger rate in the high luminosity scenarios expected in the LHC Phase-II era. This will allow to lower the muon $p_{T}$ thresholds at L1 and HLT, thus providing sensitivities for all of those physics channels characterized by soft muon signatures. Moreover, additional hits of new detectors will improve the L1 and HLT standalone muon trigger on which the physics channels with displaced muon signature in the final state completely rely. It has been shown that additional hits from GEM 


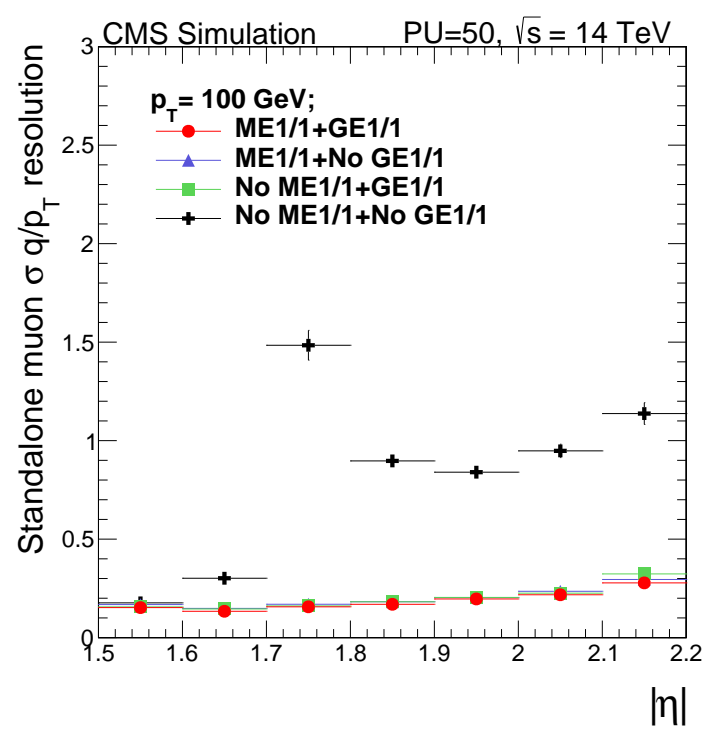

Figure 4: The dependency of the standard deviation of the $\delta\left(q / p_{T}\right) /\left(q / p_{T}\right)$ distribution as a function of the simulated muon pseudorapidity for several scenarios, illustrating the recovery of momentum resolution for standalone muons using hits in GE1/1 in the scenario where ME1/1 become non-operational due to aging or other effects. The distributions are shown for muons with $p_{T}=100 \mathrm{GeV}$ reconstructed as standalone muons in a pile-up 50 environment.

detectors, with their high spatial resolution, allows to restore the offline reconstruction efficiency and reduce the background misidentification rate, also in the high pile-up scenarios foreseen in HL-LHC era. The CMS collaboration approved the installation of the GE1/1 detector during the LS2, while the installation of the other two stations (GE2/1 and ME0) during the LS3 is still under review.

\section{Acknowledgments}

We gratefully acknowledge support from FRS-FNRS (Belgium), FWO-Flanders (Belgium), BSF-MES (Bulgaria), BMBF (Germany), DAE (India), DST (India), INFN (Italy), NRF (Korea), QNRF (Qatar), and DOE (USA).

\section{References}

[1] L. Evans and P. Bryant, LHC Machine, 3 (2008) S08001.

[2] CMS Collaboration, Technical Proposal for the Phase-II Upgrade of the Compact Muon Solenoid, June 2015, CERN-LHCC-2015-10 LHCC-P-008 CMS-TDR-15-02.

[3] CMS Collaboration, The CMS experiment at the CERN LHC, 3 (2008) S08004.

[4] F. Sauli, GEM: A new concept for electron amplification in gas detectors, Nucl. Instrum. Meth. A386 (1997) 531.

[5] CMS GEM Collaboration, CMS technical design report for the muon endcap GEM Upgrade, CERN-LHCC-2015-012, CMS-TDR-013, Jun 2015, 